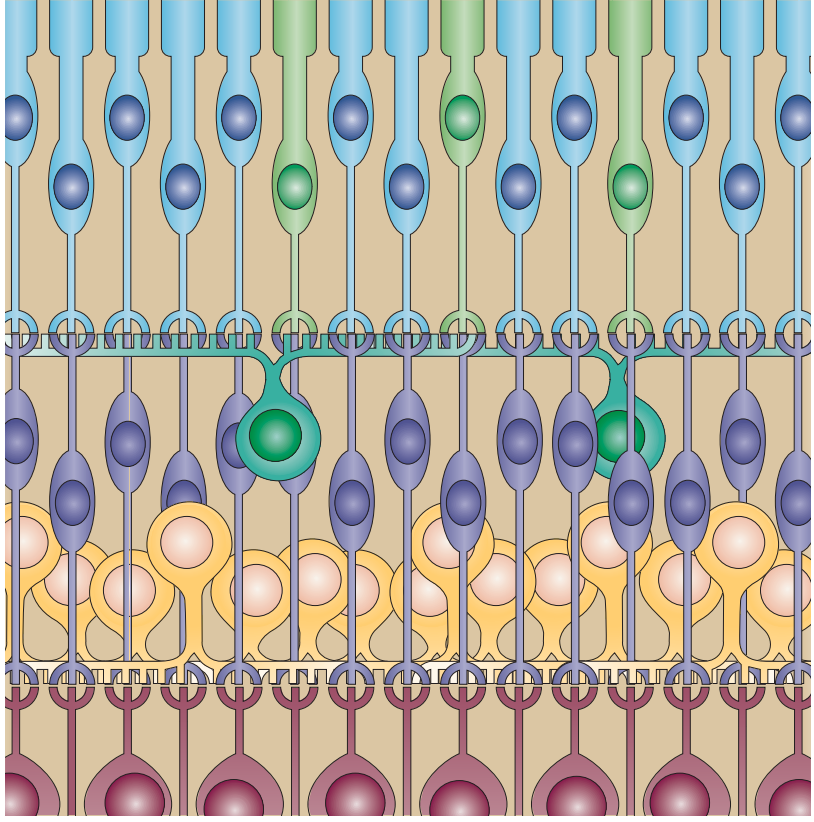

NEUROGENESIS

\section{Keeping an eye on the retina}

During retinal development in vertebrates, six types of neuron and one type of glial cell are generated in a defined temporal sequence. Two recent reports in Neuron identify transcription factors that are required for the development of three of the neuronal cell types.

In the first study, Bramblett et al. showed that the basic helix-loop-helix factor Bhlhb4 is required for the maturation but not the initial specification - of rod bipolar (RB) cells. The $\mathrm{RB}$ cells reside in the inner nuclear layer (INL) of the retina, and they relay information from rod photoreceptors to amacrine and

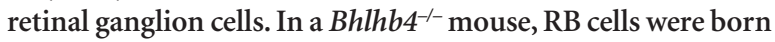
normally, but they failed to survive. The most extensive RB-cell loss was observed around postnatal day 8 , which is when Bhlhb4 expression normally peaks.

The winged helix/forkhead transcription factor Foxn 4 is expressed in progenitor cells that give rise to two other types of neuron in the INL: amacrine cells and horizontal cells. In the second study, Li et al. showed that a Foxn4 gene knockout in mice caused a profound reduction in the number of amacrine cells, and a total absence of horizontal cells. Foxn 4 seems to control amacrine cell development by regulating the expression of the bHLH factors Math3 and NeuroD1, and is both necessary and sufficient to promote an amacrine cell fate. With regard to the horizontal cells, Foxn 4 seems to have a permissive rather than an instructive role: it can make progenitor cells competent to generate horizontal cells, but cannot promote a horizontal-cell fate on its own.

Until recently, the molecular basis of retinal neurogenesis was poorly understood, but findings such as these will undoubtedly enhance our knowledge of the mechanisms that generate and maintain the diverse range of cells that coordinate retinal function.

(2) References and links

Heather Wood

ORIGINAL RESEARCH PAPERS Bramblett, D. E. et al. The transcription factor Bhlhb4 is required for rod bipolar cell maturation. Neuron 43, 779-793 (2004) | Li, S. et al. Foxn4 controls the genesis of amacrine and horizontal cells by retinal progenitors. Neuron $\mathbf{4 3}$ 795-807 (2004)

FURTHER READING Livesey, F. J. \& Cepko, C. L. Vertebrate neural cell-fate determination: lessons from the retina. Nature Rev. Neurosci. 2, 109-118 (2001)

\title{
Feeling the cold
}

The transient receptor potential (TRP) ion channels are a family of channels expressed in many cell and tissue types that allow the influx of calcium ions into cells. Little is known about their physiological function, although a role in the detection or transduction of sensory stimuli, including changes in temperature, has been suggested. Reporting in Neuron, Chuang et al. show that for some TRP channels to be maximally effective, two or more physiological stimuli must be present at the same time, indicating that they function as coincidence detectors.

One TRP channel, TRPM8, is sensitive to a reduction in ambient temperature. In response to cold, TRPM8 depolarizes afferent nerve fibres in the somatosensory system, which induces psychophysical sensations of cold. TRPM8 channels are also gated by natural cooling agents, such as menthol, and by synthetic 'super-cooling agents', such as icilin, which is not structurally related to menthol. These agonists modulate the temperature threshold at which TRPM8 channels can be activated, so that they open at higher than normal temperatures.

However, it seems that these two cooling agents work by different mechanisms. TRPM8 is rapidly activated in response to the single stimulus

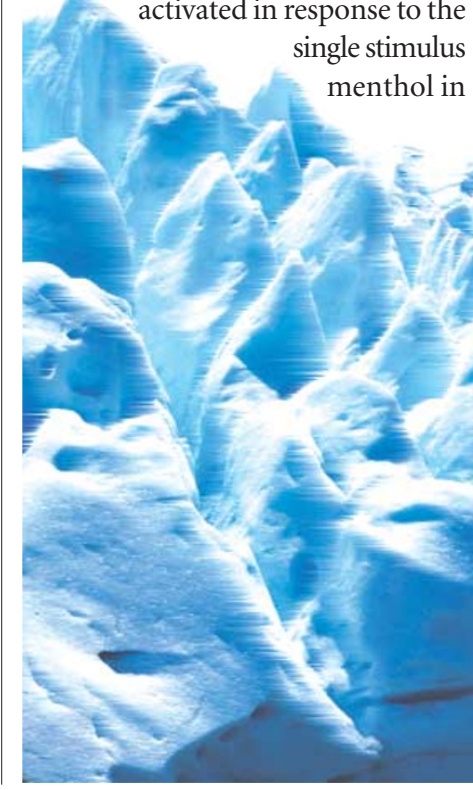

the absence of calcium ions. By contrast, as Chuang et al. show using electrophysiological techniques, full activation of TRPM8 channels by icilin occurs only with simultaneous exposure to intracellular calcium ions. Calcium ions alone apparently have no such effect on TRPM8 channels, and work only in conjunction with icilin.

The need for two independent agonists to be present simultaneously for full activation of TRPM8 channels, and their permeability to calcium ions, could allow TRPM8 to modulate its gating activity according to the concentration of icilin or similar agonists and the presence of another stimulus that promotes an increase in intracellular calcium. This, in turn, might allow cells to fine-tune their sensitivity to a specific chemical stimulus and to increase the range of environmental signals to which they are sensitive - a property that is especially useful for channels involved in the transduction of sensory stimuli.

Alison Rowan

(2) References and links ORIGINAL RESEARCH PAPER Chuang, H.-h., Neuhausser, W. M. \& Julius, D. The super-cooling agent icilin reveals a mechanism of coincidence detection by a temperature-sensitive TRP channel. Neuron 43, 859-869 (2004)

FURTHER READING Patapoutian, A., Peier, A. M., Story, G. M. \& Viswanath, V. ThermoTRP channels and beyond: mechanisms of temperature sensation. Nature Rev. Neurosci. 4 , 529-539 (2003)

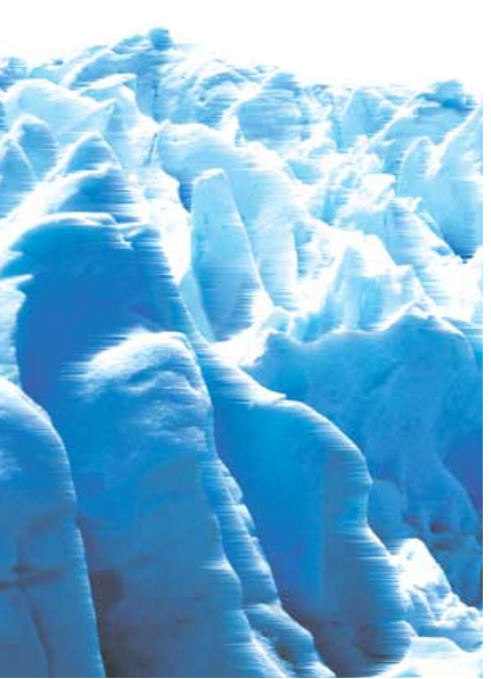

